C-A/AP/\#214 July 2005

\title{
Simulation Calculations and Analytical Predictions of Eigenmode Projection Parameters
}

Y. Luo

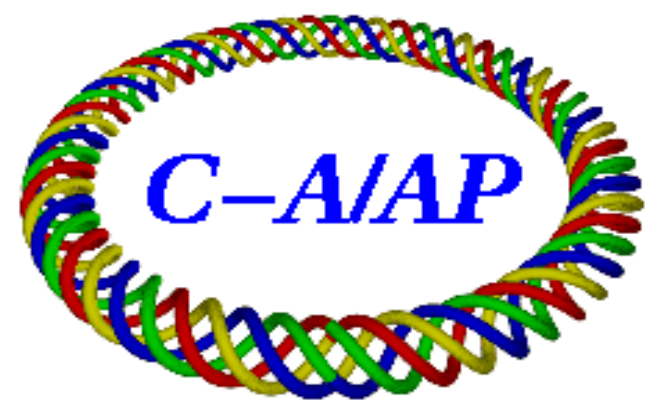

Collider-Accelerator Department

Brookhaven National Laboratory

Upton, NY 11973 
$\mathrm{CAD} / \mathrm{AP} / 214$

July 19,2005

\title{
Simulation Calculations and Analytical Predictions of Eigenmode Projection Parameters
}

\author{
Y. Luo \\ Brookhaven National Laboratory, Upton, NY 11973, USA
}

In the article, we carry out the element-by-element tracking simulations with the Relativistic Heavy Ion Collider (RHIC) Blue ring lattice to numerically calculate the eigenmode projection parameters. Eigenmode projection parameters are being measured and tested in RHIC for the possible future continuous global coupling measurement and correction. The simulation calculations and analytical predictions prove that the local eigenmode projection measurement does hint the global coupling coefficient, including its amplitude and phase. Therefore, to determine the global coupling, one observation point in the ring is sufficient. And comparisons between the simulation calculations and the analytical predications from different linear weak coupling approaches are also presented.

\section{Eigenmode Projections}

For the linearly coupled optics, $x$ and $y$ coordinates of the free oscillation can be cast as

$$
\left\{\begin{array}{l}
x_{n}=A_{I, x} \cos \left[2 \pi Q_{1}(n-1)+\phi_{I, x}\right]+A_{I I, x} \cos \left[2 \pi Q_{2}(n-1)+\phi_{I I, x}\right] \\
y_{n}=A_{I, y} \cos \left[2 \pi Q_{1}(n-1)+\phi_{I, y}\right]+A_{I I, y} \cos \left[2 \pi Q_{2}(n-1)+\phi_{I I, y}\right]
\end{array} .\right.
$$

In the following, we assume that eigenmode I is more linked to the horizontal plane, and eigenmode II is more linked to the vertical plane. $Q_{I}$ and $Q_{I I}$ are the two eigentunes. $A_{i, z}, i=I, I I, z=x, y$, is the amplitude of the eigenmode $i$ 's projection onto the $z$ axis. They are non-negative numbers. $\phi_{i, z}$ is the phase of the eigenmode $i$ 's projection onto the the $z$ axis. $A_{i, z}$ and $\phi_{i, z}$ actually are the eigenmode projection parameters. They define the eigenmode projection ellipses in the $(x-y)$ plane.

Besides the two eigentunes $Q_{I}$ and $Q_{I I}$, we define another four weak difference coupling observables [1]. $R_{I}$ and $R_{I I}$ are the eigenmode projection amplitude ratios,

$$
\left\{\begin{array}{c}
R_{I}=\frac{A_{I, y}}{A_{I, x}} \\
R_{I I}=\frac{A_{I I, x}}{A_{I I, y}}
\end{array} .\right.
$$

$R_{i}$ is linked to eigenmode $i$. Under the uncoupled situation, $R_{I, I I}=0$. And we define $\Delta \phi_{I, I I}$ as the eigenmode projection phase differences,

$$
\left\{\begin{array}{ccc}
\Delta \phi_{I} & =\phi_{I, y}-\phi_{I, x} \\
\Delta \phi_{I I} & = & \phi_{I I, x}-\phi_{I I, y}
\end{array} .\right.
$$

$\Delta \phi_{i}$ is linked to eigenmode $i$. Under the uncoupled situation, $\Delta \phi_{I}$ and $\Delta \phi_{I I}$ have no meaning.

The fractional eigentune split $\left|Q_{I}-Q_{I I}-p\right|$, where $p$ is the integer tune split, has been conventionally used as the coupling observable in the skew quadrupole scan decoupling. And it is also adopted in the novel skew quadrupole modulations $[2,3,4,5]$. There, to obtain the residual coupling from the eigentune split, we have to scan or modulate skew quadrupoles.

With the high resolution phase locked loop (PLL) tune measurement system, the above six coupling observables can be continuously measured [6]. They define the local coupling information, the eigenmode projection ellipses in $(x-y)$ plane, according to Eq. (1). From their analytical calculations, we found they also tell the global coupling coefficient, shown in the Appendix.

In the following, we carry out the element-by-element tracking simulations with the Relativistic Heavy Ion Collider (RHIC) Blue ring lattice to numerically calculate the eigenmode projection parameters. Compar-

isons between the simulation calculations and the analytical predications from different linear weak coupling approaches are also presented. 


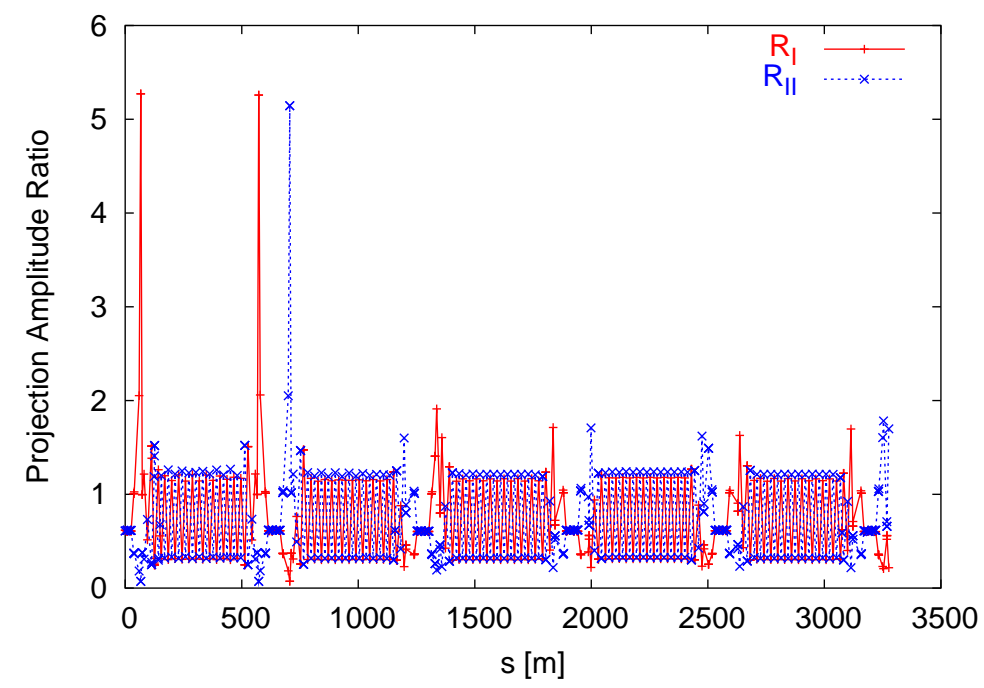

Figure 1: $R_{I}$ and $R_{I I}$ along the ring.

\section{Simulation calculations and analytical predictions}

In this section, we carry out the element-by-element simulation with the RHIC Blue ring lattice to numerically calculate the above defined four eigenmode projections, $R_{I, I I}$ and $\Delta \phi_{I, I I}$.

A single particle with non-zero initial position coordinates is launched at IP6. 4096 turn's simulation tracking is performed with the SAD code. The turn-by-turn (TBT) $(x, y)$ data are recorded at each BPM or markers. According to Eq. (1), the eigenmode's projection parameters $A_{i, z}$ and $\phi_{i, z}$ are calculated with the fast Fourier transformation (FFT) technique.

Each RHIC ring has three correction skew quadrupole families. Each family has 16 skew quadrupole magnets. As an example, in the section we set the uncoupled tunes $\left(Q_{x, 0}, Q_{y, 0}\right)=(28.22,29.23)$. We artificially add coupling sources into the originally uncoupled optics model by setting skew quadrupole families $\mathrm{F} 1$ and $\mathrm{F} 3$ to $\left(k_{s} d l\right)_{1,3}=0.0002 \mathrm{~m}^{-1}$.

\subsection{Simulation calculations from the element-by-element tracking}

Fig. 1 and Fig. 2 shows $R_{I}$ and $R_{I I}$ along the ring and in the first $700 \mathrm{~m}$ from IP6. Fig. 3 shows $R_{I} R_{I I}$ along the ring. Fig. 4 and Fig. 5 show $\tan \left(\Delta \phi_{I, I I}\right)$ along the ring and in the first $700 \mathrm{~m}$ from IP6.

According to Fig. 1 and Fig. 2, the eigenmode projection's amplitude ratios vary along the ring. However, it is noticed from Fig. 4 that the variation of $R_{I} R_{I I}$ is very small,

$$
\left\{\begin{array}{c}
\left(R_{I} R_{I I}\right)_{\text {mean }}=0.3738 \\
\left(R_{I} R_{I I}\right)_{\text {rms }}=0.0053
\end{array},\right.
$$

The rms value is about $1.4 \%$ of the mean value.

\subsection{Analytical predications of projection amplitude ratios}

\subsection{1 from Hamiltonian perturbation approach}

According to weak linear difference coupling's Hamiltonian approach, $R_{I} R_{I I}$ is constant along the ring, as given by Eq. (19). $R_{I} R_{I I}$ is decided by the uncoupled tune split $\Delta$ and the amplitude of the defined coupling coefficient $\left|C^{-}\right|$. According to Hamiltonian perturbation approach, for the above lattice,

$$
R_{I} R_{I I}=0.3597,
$$

which is about $3.7 \%$ away from the calculated $\left\langle R_{I} R_{I I}\right\rangle$ from the above simulation. Hamiltonian perturbation approach predicts $\left\langle R_{I} R_{I I}\right\rangle$ very well. However, it fails in giving the predications to the samll variations in $R_{I} R_{I I}$ along the ring. 


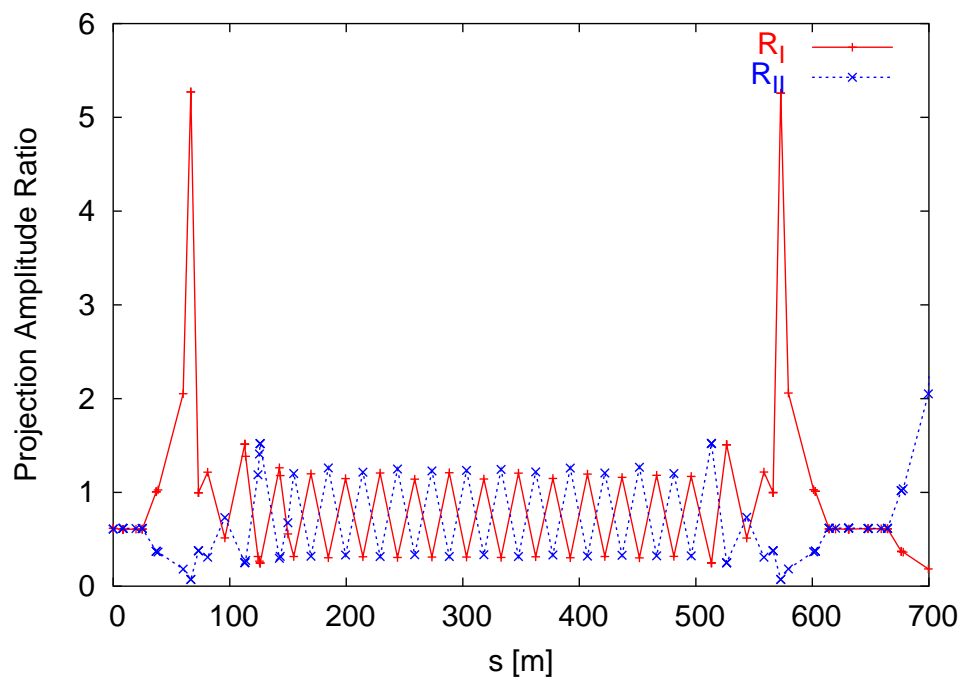

Figure 2: $R_{I}$ and $R_{I I}$ in the first $700 \mathrm{~m}$.

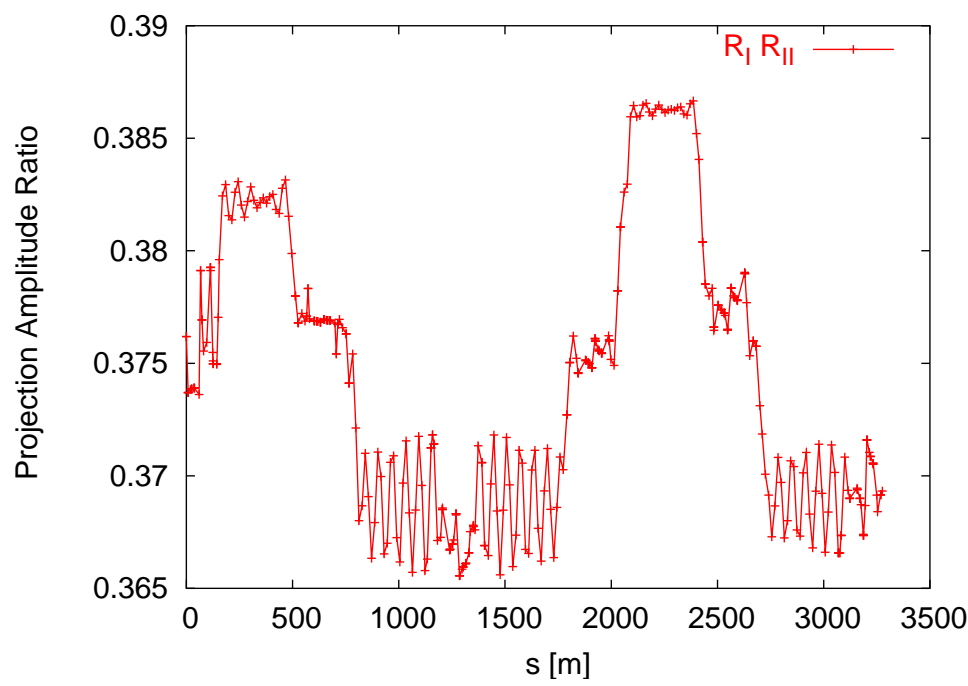

Figure 3: $R_{I} R_{I I}$ along the ring.

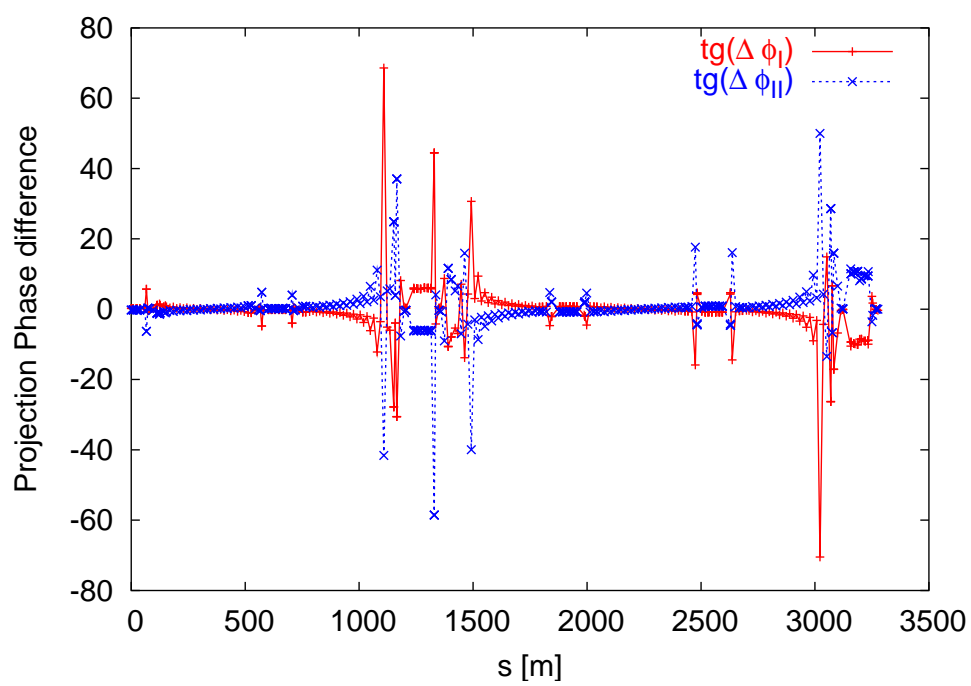

Figure 4: $\tan \left(\Delta \phi_{I, I I}\right)$ along the ring. 


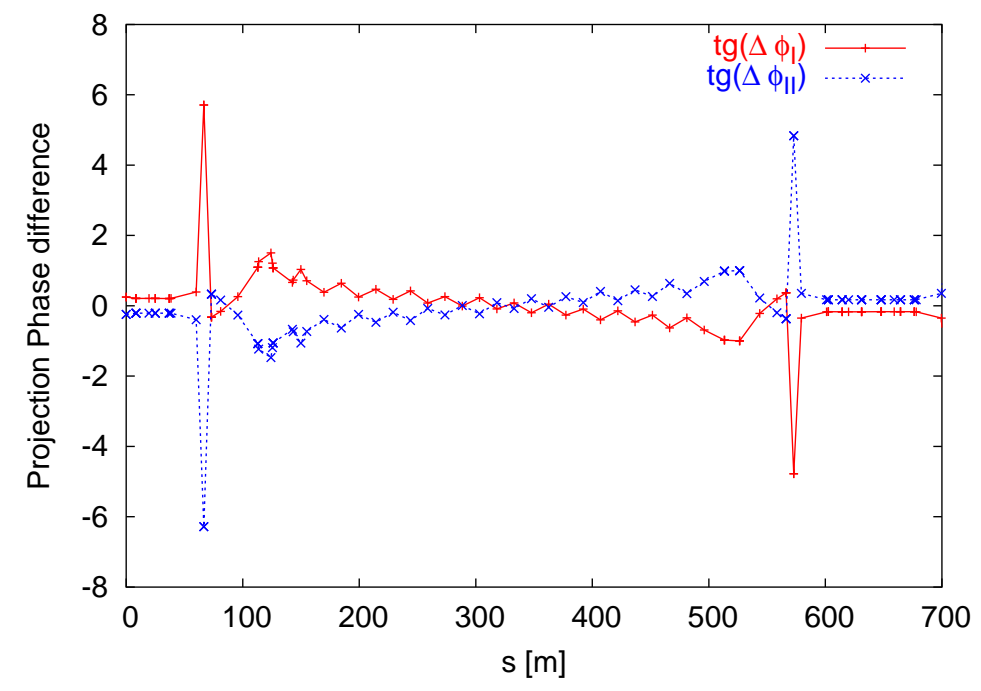

Figure 5: $\tan \left(\Delta \phi_{I, I I}\right)$ in the first $700 \mathrm{~m}$.

\subsection{2 from matrix perturbation approach}

According to the linear weak difference coupling's matrix perturbation approach, $R_{I} R_{I I}$ is decided by $r$, as given by Eq. (35). $r$ is calculated according to Eq. (32). Fig. 6 shows the calculated $r$ from the matrix perturbation approach and the strict matrix approach. The strict matrix approach is based on the strict linear coupling's action-angle parameterization. The strict matrix approach gives $r=0.8532 \pm 0.001542$, the matrix perturbation approach gives $r=0.8525 \pm 0.0003168$. They agree very well in the averaged $r$. And they show the $r$ jumps at the same locations. $r$ jump happens at the individual coupling sources.

Fig. 7 shows $R_{I} R_{I I}$ along the ring calculated according to Eq. (32), together with these from the simulation calculation. The analytical prediction from the matrix perturbation approach gives

$$
\left\{\begin{array}{ccc}
\left(R_{I} R_{I I}\right)_{\text {mean }} & =0.3760 \\
\left(R_{I} R_{I I}\right)_{\text {rms }} & =0.00102
\end{array} .\right.
$$

The mean value is $0.59 \%$ away from the simulation calculation.

\subsection{3 from strict matrix approach}

Fig. 8 shows $R_{I} R_{I I}$ calculated from the strict matrix approach Eq. (43), together with these from the simulation calculation. It gives

$$
\left\{\begin{array}{c}
\left(R_{I} R_{I I}\right)_{\text {mean }}=0.3737 \\
\left(R_{I} R_{I I}\right)_{\text {rms }}=0.0053
\end{array} .\right.
$$

The predictions of $R_{I} R_{I I}$ agree very well with the simulation calculations along the ring, even at local observation points. .

\subsection{Analytical predications of projection phase differences}

Both of Hamiltonian and matrix perturbation approaches predict the projection phase differences. From the perturbation approaches,

$$
\tan \Delta \phi_{I}=-\tan \Delta \phi_{I I}
$$

This holds very well in the simulation calculation result, as shown in Fig. 4 and Fig. 5.

Fig. 9 shows the predictions of the phase differences calculated from $C^{-}$in the Hamiltonian perturbation approach. Fig. 10 shows the predictions of the phase differences calculated from $h_{-}$in matrix perturbation approach. Both agree very well with the simulation results, except at several points. It is noticed that phases of $C^{-}$and $h_{-}$have opposite signs. Fig. 11 gives the phase differences calculated from strict matrix approach according to Eq. (44). 


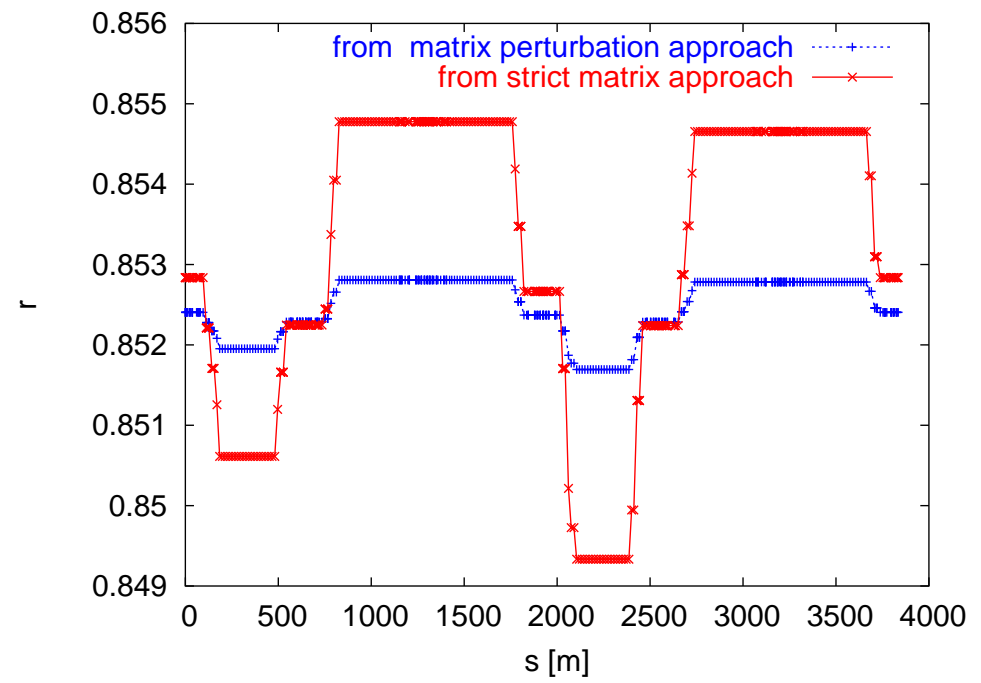

Figure 6: $r$ from the strict and perturbation matrix approaches.

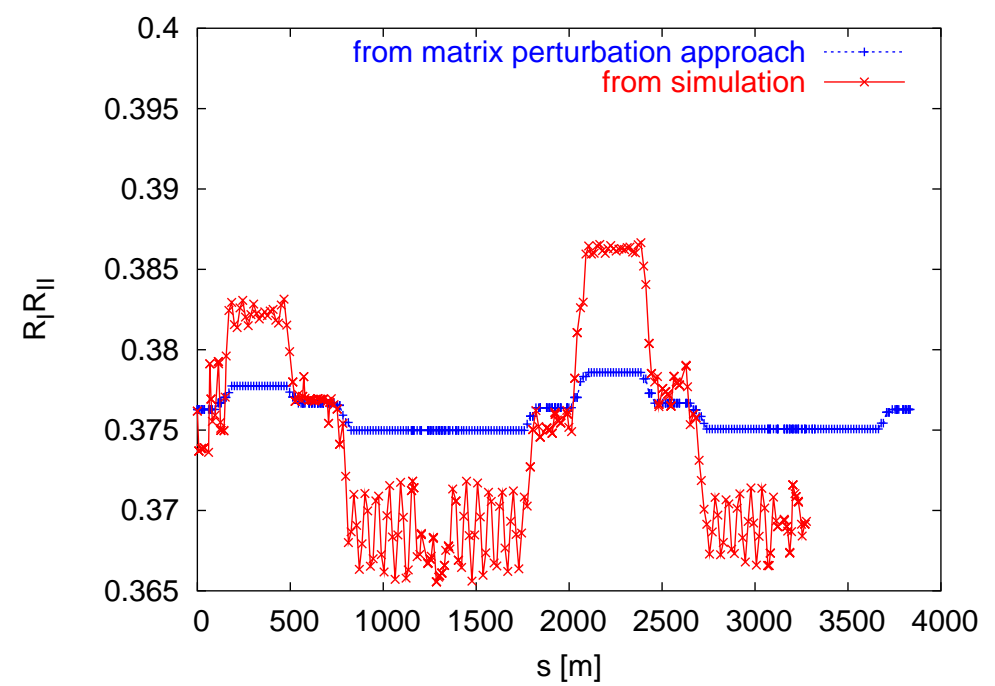

Figure 7: $R_{I} R_{I I}$ calculated from perturbation matrix approach.

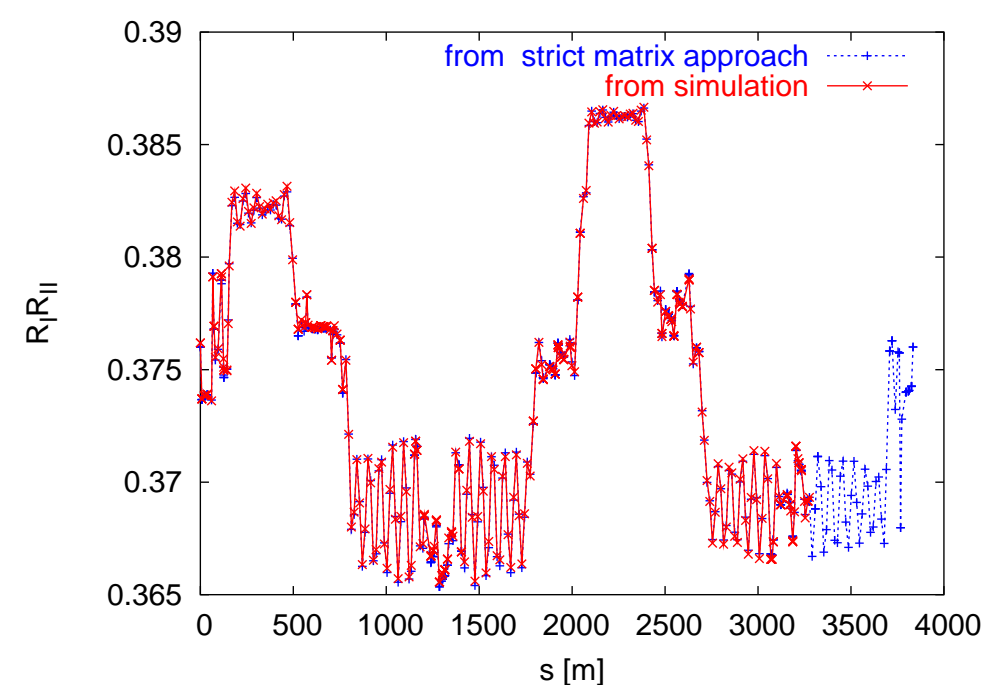

Figure 8: $R_{I} R_{I I}$ calculated from strict matrix approach. 


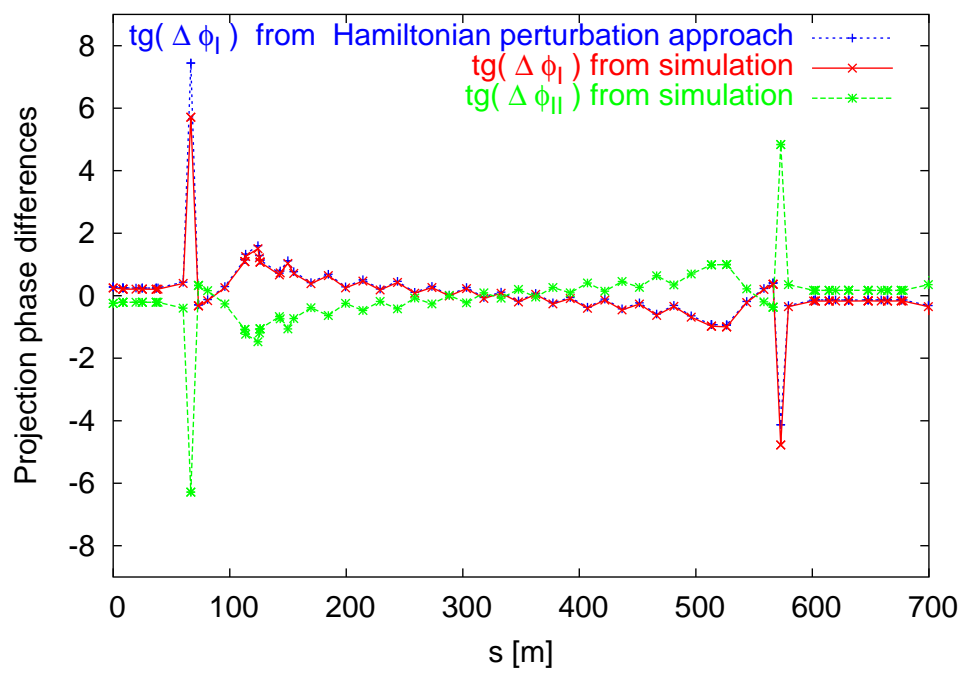

Figure 9: Phase difference calculated from Hamiltonian perturbation approach.

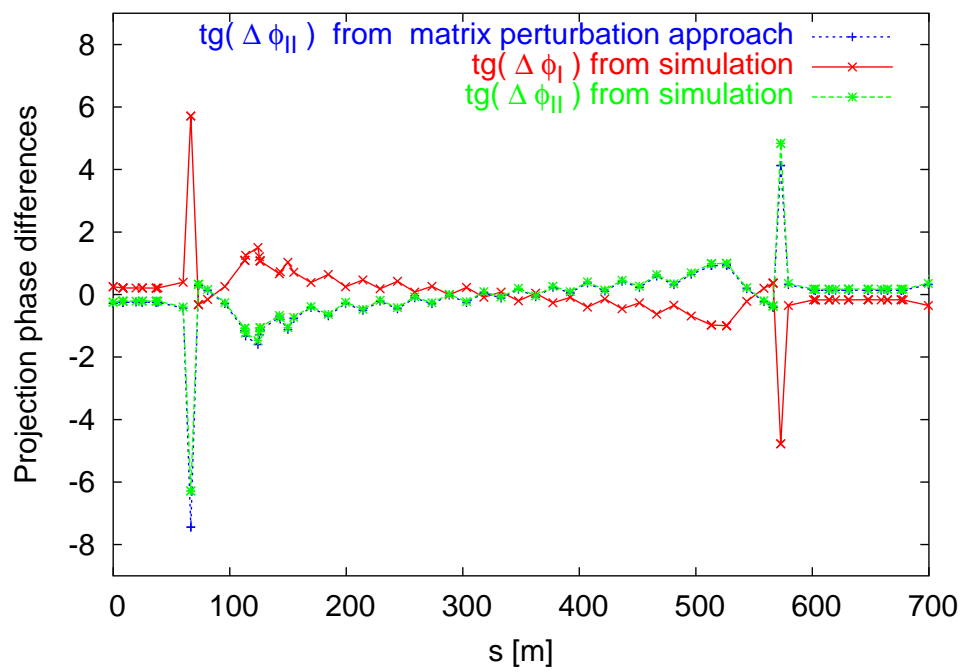

Figure 10: Phase difference calculated from matrix perturbation approach.

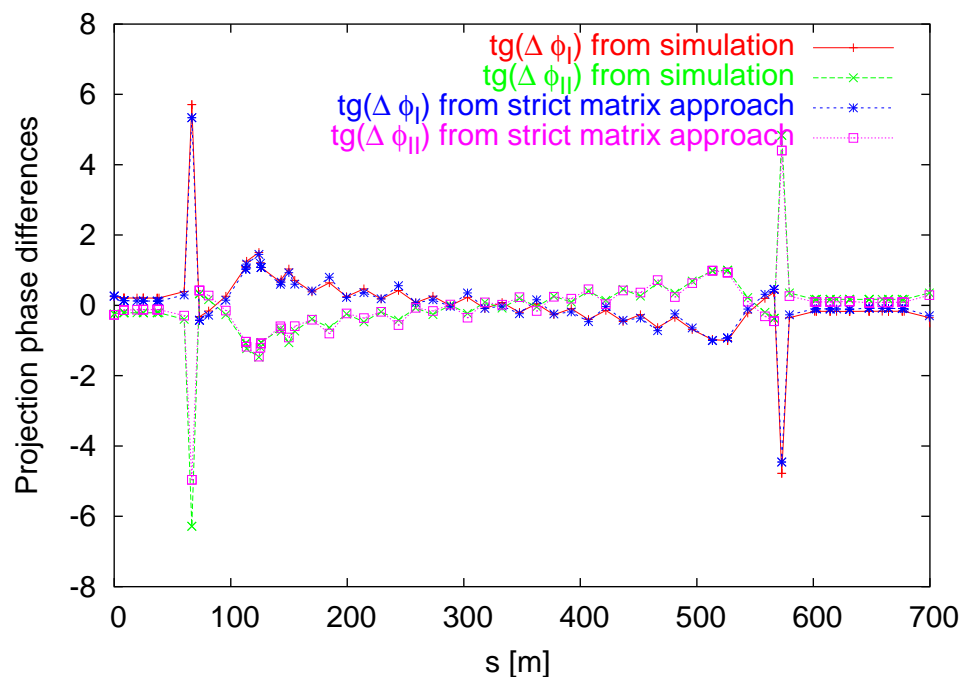

Figure 11: Phase difference calculated from strict matrix approach. 


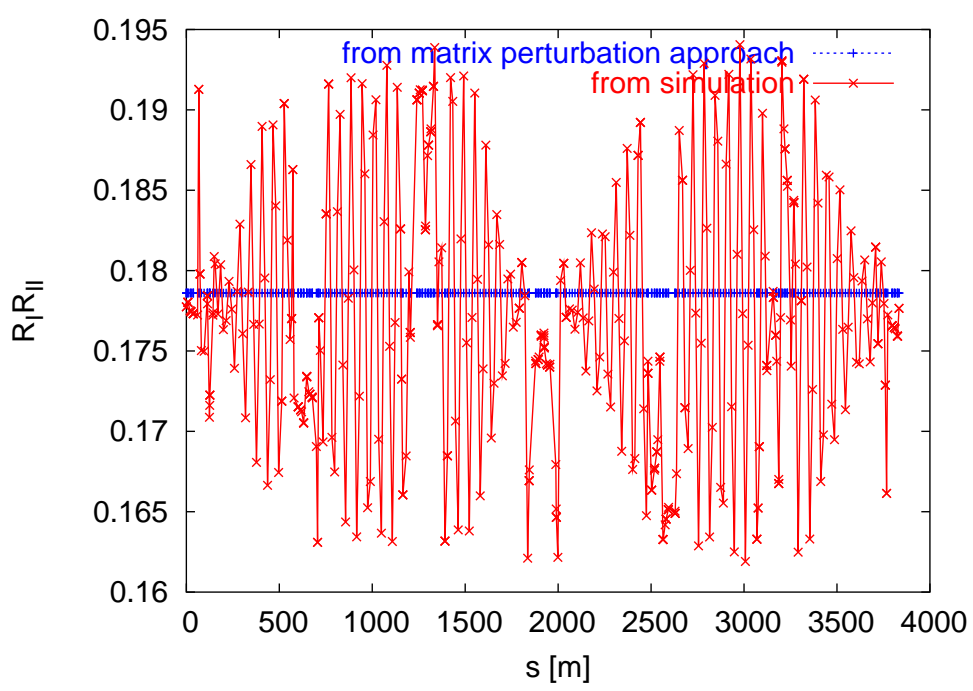

Figure 12: $R_{I} R_{I I}$ from the matrix perturbation approach and from the simulation calculation. There is only one coupler in the uncoupled optics.

\subsection{Summary}

The simulation calculations and analytical predictions prove that the local eigenmode projection measurement does hint the global coupling coefficient, including its amplitude and phase. Hamiltonian perturbation and matrix perturbation approaches give very good prediction of the mean value of $R_{I} R_{I I}$ along the ring. $<R_{I} R_{I I}>$ tells the global coupling strength. The variations in $R_{I} R_{I I}$ is due to the distribution of the couplers. The rms value of $R_{I} R_{I I}$ along the ring is much smaller than the mean value.

Both of Hamiltonian and matrix perturbation approaches predict the projection phase differences well. They tell the phase of the coupling coefficient $C^{-}$in the Hamiltonian perturbation approach, or $h_{-}$in the matrix perturbation approach. The phases of $C^{-}$and $h_{-}$changes along the ring. However, the phase differences at different observation points are related, as shown by Eq. (12).

\section{Other interested examples}

In this section, we give the $R_{I} R_{I I} \mathrm{~s}$ from different simulation lattices. The predications from the matrix perturbation and strict matrix approaches are also given.

\subsection{Only one coupler in the ring}

We add only one coupler SQSKLC6 into the uncoupled optics model. The uncoupled tunes are $\left(Q_{x, 0}, Q_{y, 0}\right)=$ $(28.22,29.23)$, SQSKLC6 's strength is set to $\left(k_{s} d l\right)=0.003 \mathrm{~m}^{-1}$.

Fig. 12 shows $R_{I} R_{I I}$ along the ring from the matrix perturbation approach and from the simulation calculation. From matrix perturbation approach, $R_{I} R_{I I}$ should be constant along the ring. However, from the element-by-element tracking simulation, $R_{I} R_{I I}$ oscillates. And the rms value is not small comparing with the mean value.

Fig. 13 shows $R_{I} R_{I I}$ along the ring from the strict matrix approach and from the simulation calculation. They agree very well along the ring.

\subsection{Only two couplers in the ring}

Here we add two couplers SQSKLC6 and SQSKLC8 into the uncoupled optics. Their strengths are set to $\left(k_{s} d l\right)=0.003 \mathrm{~m}^{-1}$.

Fig. 14 shows $R_{I} R_{I I}$ along the ring from the matrix perturbation approach and from the simulation calculation. From matrix perturbation approach, there are two jumps in $R_{I} R_{I I}$ at the locations of the two couplers. From the element-by-element tracking simulation calculation, the jumps also show up. However, between the two predicted jumps, $R_{I} R_{I I}$ oscillates. 


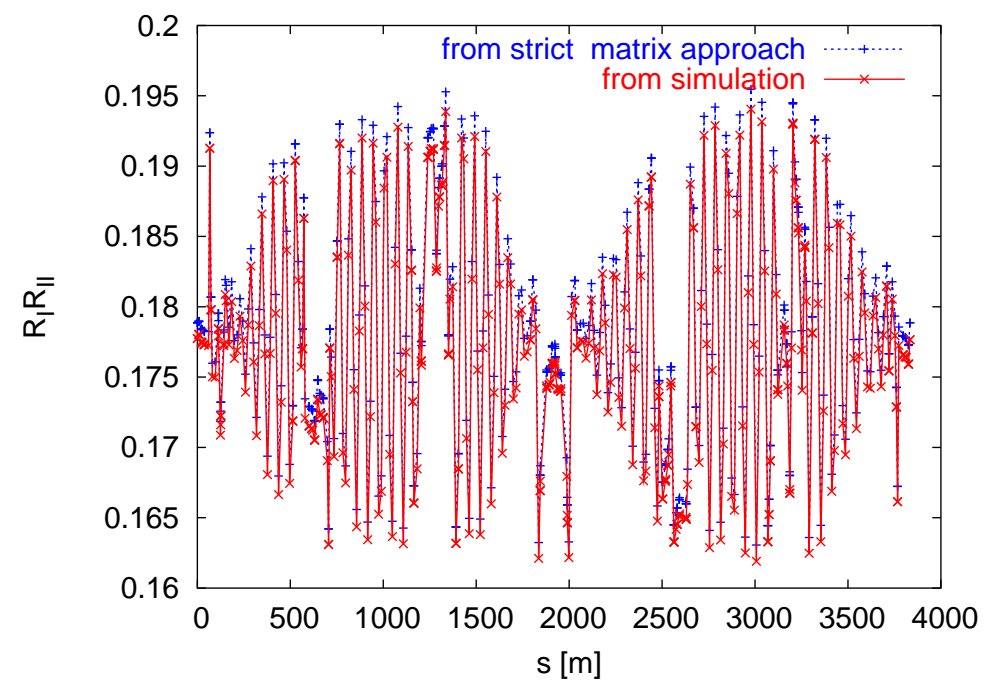

Figure 13: $R_{I} R_{I I}$ from the strict matrix approach and from the simulation calculation. There is only one coupler in the uncoupled optics.

Fig. 15 shows $R_{I} R_{I I}$ along the ring from the strict matrix approach and from the simulation calculation approach. The $R_{I} R_{I I}$ shapes along the ring are very similar. It not clear why $R_{I} R_{I I}$ s from the strict matrix approach are 0.01 larger than these from the simulation calculation.

\subsection{Only four couplers in the ring}

Then we add four couplers into the uncoupled optics. They are SQSKLC6, SQSKLC8, SQSKLC12, and SQSKLC2. Their strengths are set to $\left(k_{s} d l\right)=0.003 \mathrm{~m}^{-1}$. These four couplers' contributions to the global coupling coefficient $C^{-}$cancel each other.

Fig. 16 shows $R_{I} R_{I I}$ along the ring from the matrix perturbation approach and from the simulation calculation. The average of $R_{I} R_{I I}$ is very small, which is due to the weak global coupling. From matrix perturbation approach, there should be four jumps in $R_{I} R_{I I}$ along the ring. However, from the element-byelement tracking simulation calculation, these predicted jumps are not apparent.

Fig. 17 shows $R_{I} R_{I I}$ along the ring from the strict matrix approach and from the simulation calculation. The predictions from the strict matrix approach agree very well with the simulation calculations.

\subsection{Summary}

We compared the simulation calculations of $R_{I} R_{I I}$ with the predications from the matrix perturbation and strict matrix approaches. Matrix perturbation approach predicts $<R_{I} R_{I I}>$ very well. However, the predicted jumps in $R_{I} R_{I I}$ at the locations of the couplers are not verified with the simulation calculations. Strict matrix approach always gives very good predictions to $R_{I} R_{I I}$ along the ring under different simulation lattices.

\section{$4 \quad R_{I} R_{I I}$ versus the uncoupled tune split}

Here we calculate $R_{I} R_{I I}$ versus the uncoupled tune split $\Delta$. From the Hamiltonian and matrix perturbation approaches, when the uncoupled tune split $\Delta=0, R_{I} R_{I I}=1$. This feature is used for PLL measured $R_{I} R_{I I}$ calibration. Fig. 18 shows the amplitude ratios versus the uncoupled tune split. The optics is the same as that in section 2 . To get better calibration, the design tunesplit should be reached below 0.001 . 


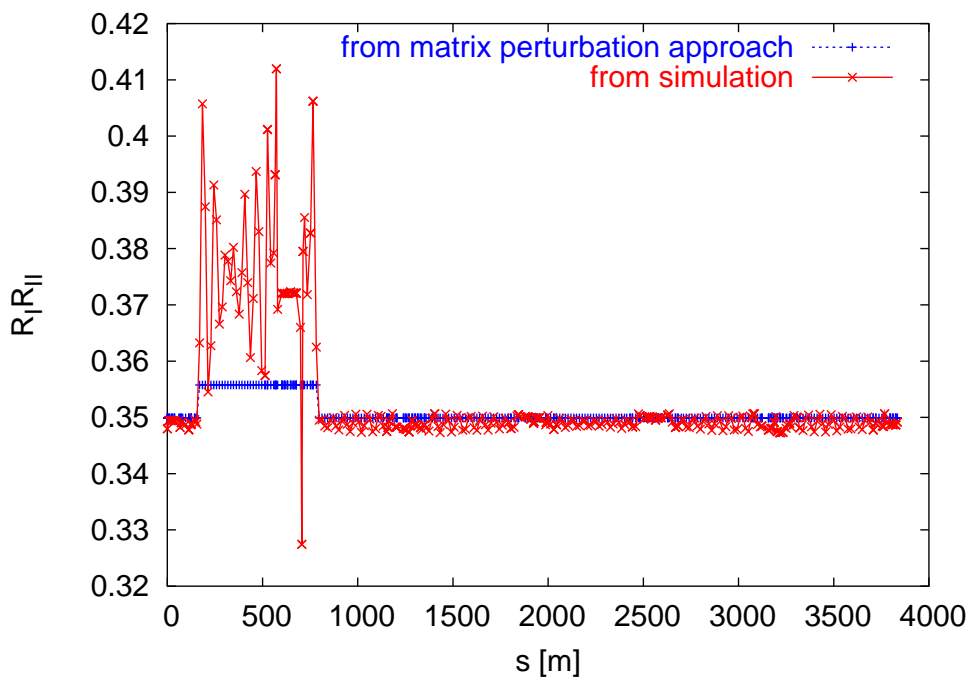

Figure 14: $R_{I} R_{I I}$ from the matrix perturbation approach and from the simulation calculation. There are only two couplers in the uncoupled optics.

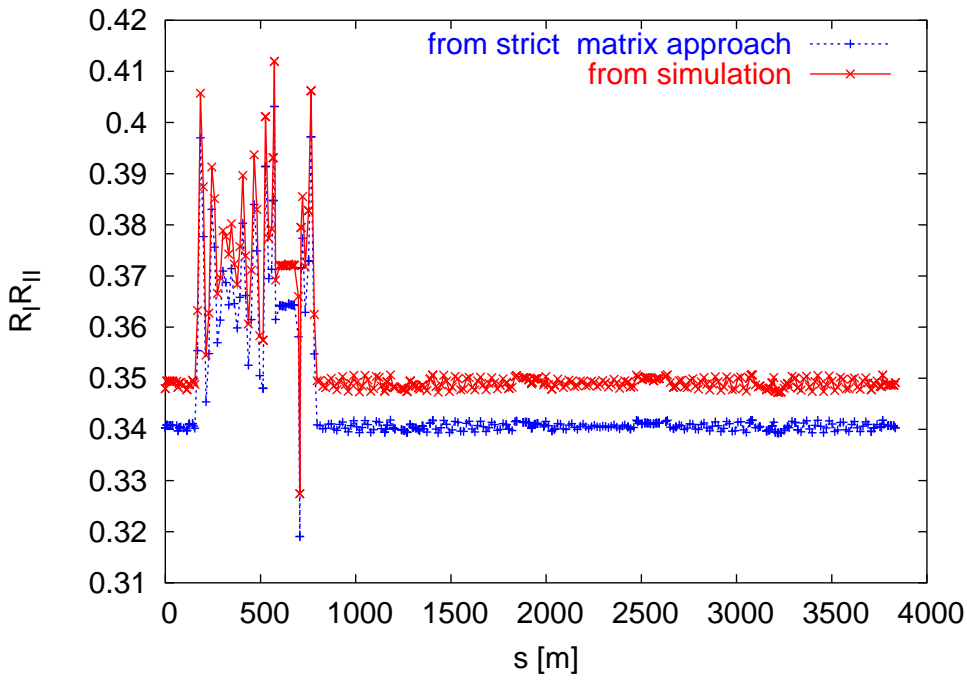

Figure 15: $R_{I} R_{I I}$ from the strict matrix approach and from the simulation calculation. There are only two couplers in the uncoupled optics. 


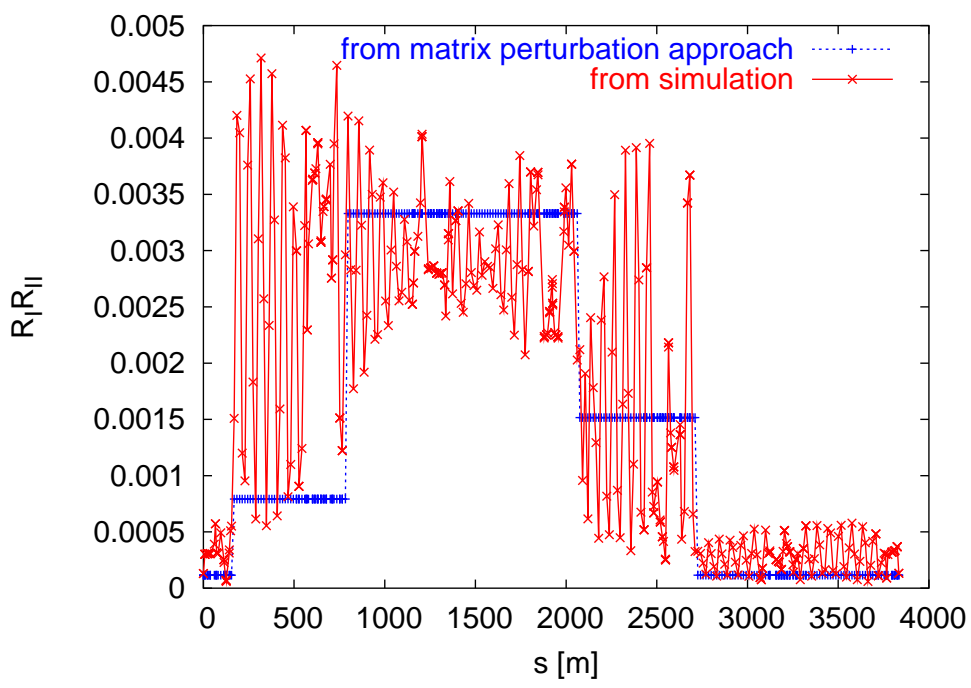

Figure 16: $R_{I} R_{I I}$ from the matrix perturbation approach and from the simulation calculation. There are only four couplers in the uncoupled optics.

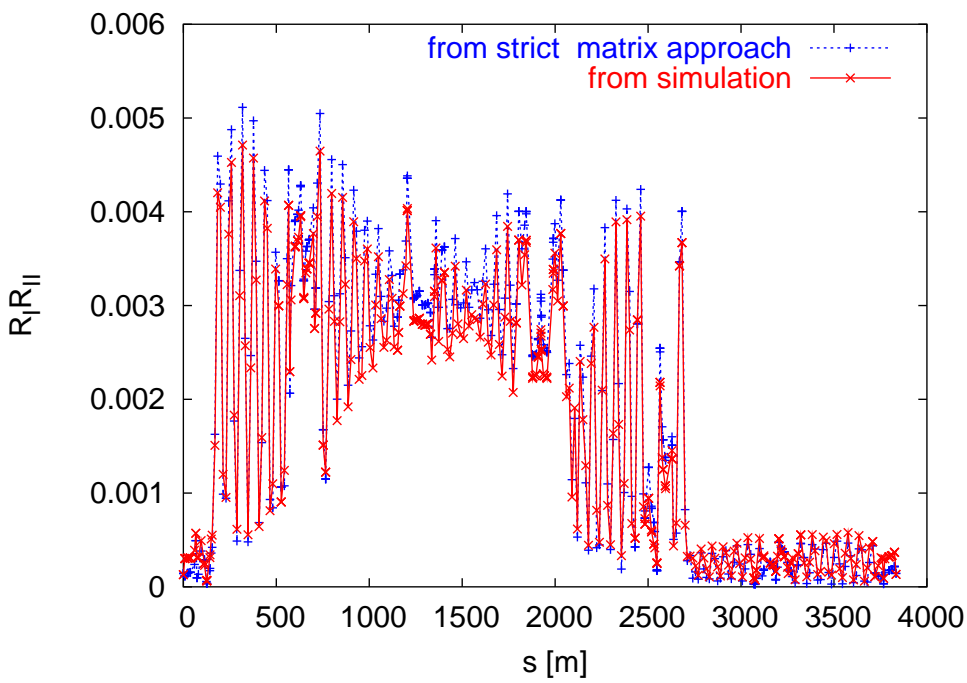

Figure 17: $R_{I} R_{I I}$ from the strict matrix approach and from the simulation calculation. There are only four couplers in the uncoupled optics. 


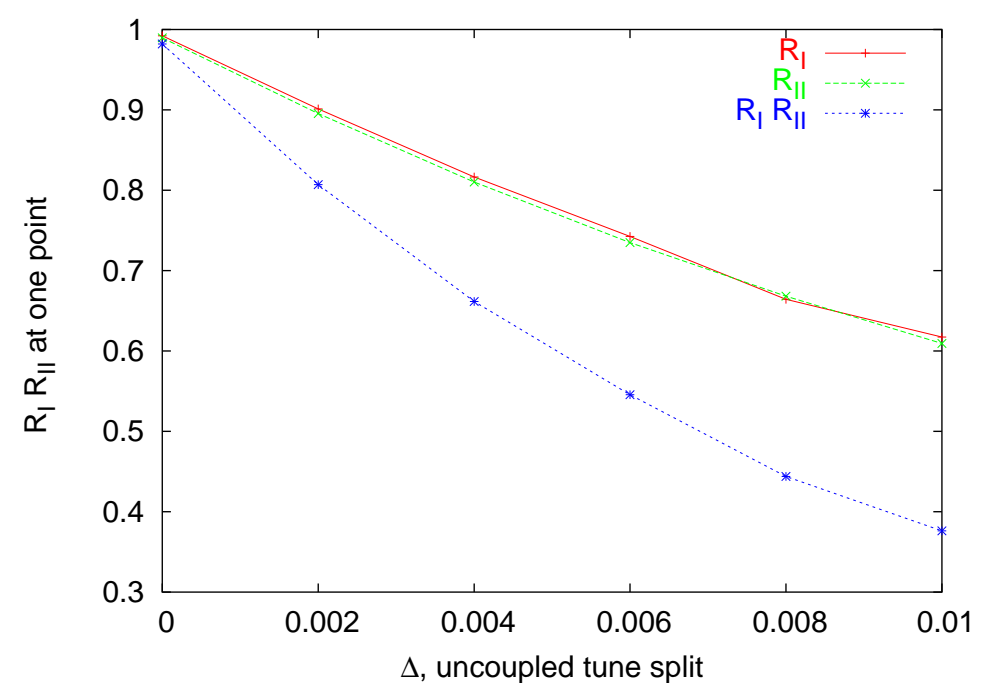

Figure 18: $c_{11}$ comparison along the ring.

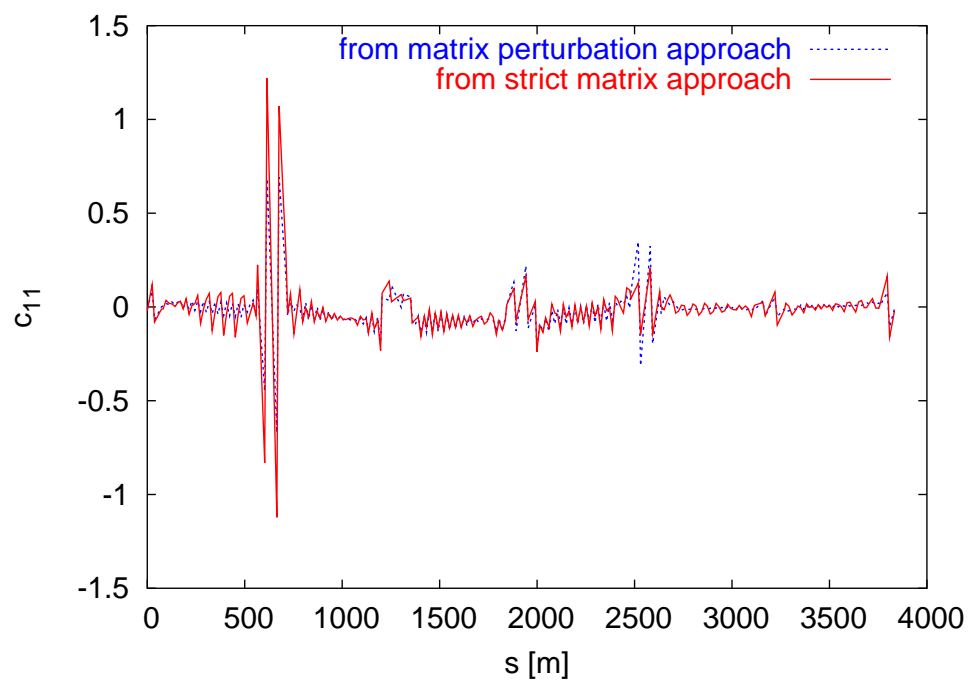

Figure 19: $c_{11}$ predication from matrix perturbation approach.

\section{Predication of $\mathrm{C}$ from matrix perturbation approach}

Here we compare the elements of coupling matrix $\mathbf{C}$ between the strict matrix approach and the matrix perturbation approach. According to weak linear difference coupling's matrix perturbation approach,

$$
\mathbf{C}=\mathbf{U}_{I} \overline{\mathbf{C}} \mathbf{U}_{I I}^{-1} .
$$

We calculate the normalized coupling matrix $\overline{\mathbf{C}}$ according to Eq. (32). In above $\mathbf{U}_{i} \mathrm{~s}$, we use uncoupled Twiss parameters.

We still assume the uncoupled tunes $\left(Q_{x, 0}, Q_{y, 0}\right)=(28.22,29.23)$ and we artificially add four couplers into the uncoupled optics. They are SQSKLC6, SQSKLC8, SQSKLC12, and SQSKLC2. Their strengths are set to $\left(k_{s} d l\right)=0.003 \mathrm{~m}^{-1}$. These four couplers' contributions to the global coupling coefficient $C^{-}$cancel each other. Fig. 19 to Fig. 22 show the comparisons of $c_{11}, c_{12}, c_{21}$, and $c_{22}$ from matrix perturbation and strict matrix approaches.

The predication accuracy of the matrix perturbation approach changes with different coupling situations. 


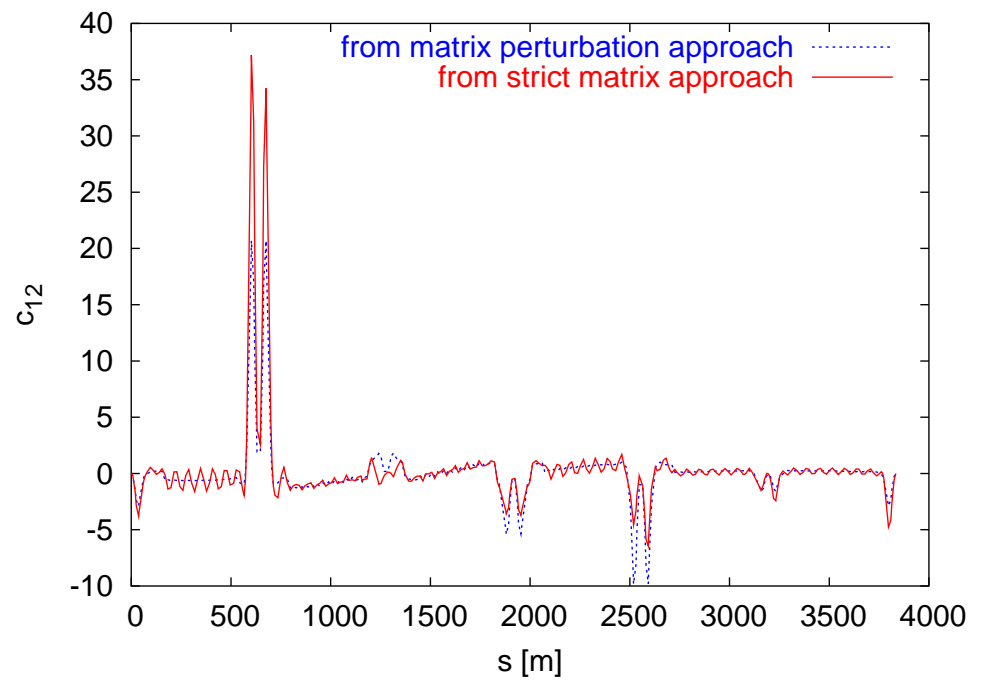

Figure 20: $c_{12}$ predication from matrix perturbation approach.

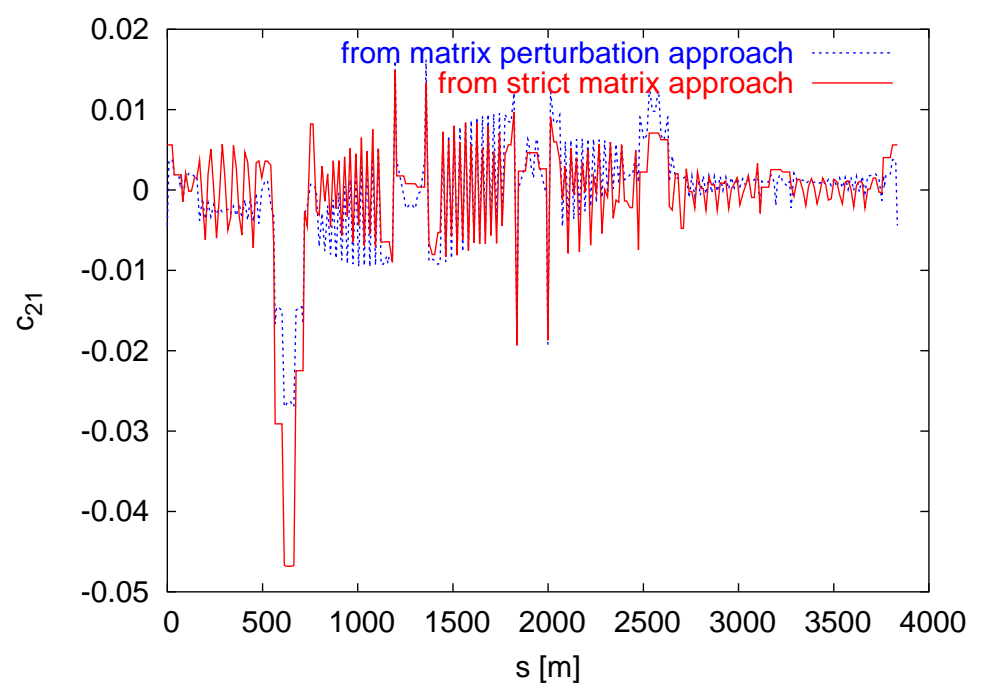

Figure 21: $c_{21}$ predication from matrix perturbation approach. .

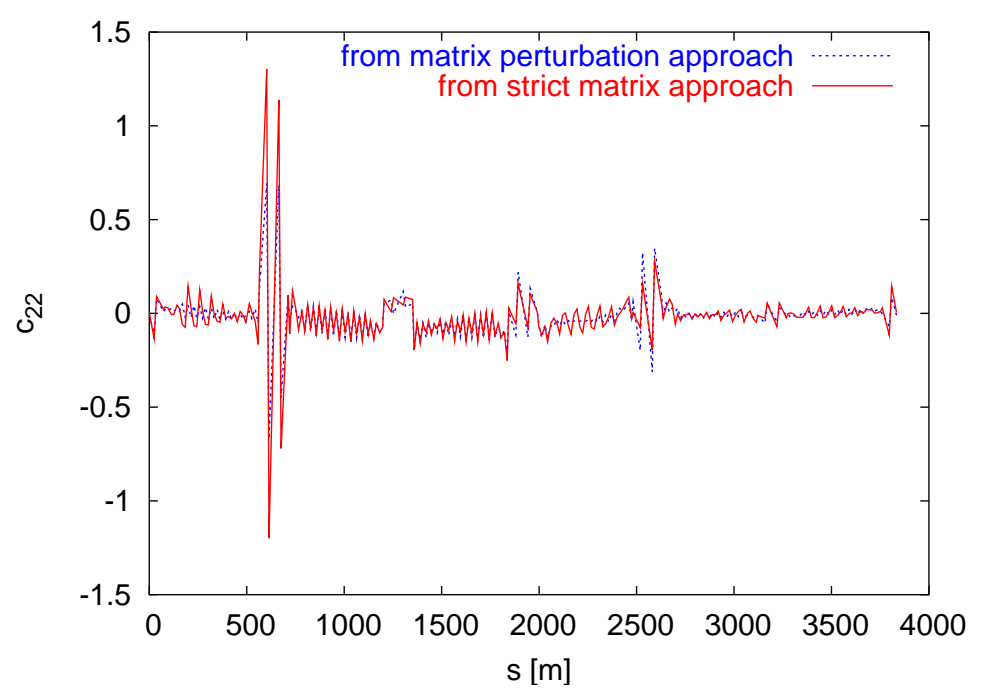

Figure 22: $c_{22}$ predication from matrix perturbation approach. . 


\section{Acknowledgments}

The authors thank T. Satogata, D. Trbojevic for discussions. This work is support by the US DOE under contract No. DE-AC02-98CH10886.

\section{References}

[1] Y. Luo, P. Cameron, S. Peggs, D. Trbojevic, Possible phase loop for the global decoupling, RHIC-AP174 .

[2] Y. Luo, et al., Phys. Rev. ST Accel. Beams 8, 014001 (2005).

[3] Y. Luo, et al., Robust and fast global decoupling with coupling angle modulation, submitted to Phys. Rev. ST Accel. Beams.

[4] Y. Luo, et al., Principles of the global decoupling on the ramp, BNL C-AD/AP Note 162, Aug., 2004, (unpublished).

[5] Y. Luo, et al., Global decoupling on the RHIC ramp, BNL C-AD/AP Note 205, May, 2005, (unpublished).

[6] Y. Luo, P. Cameron, et al., Possible phase loop for the global decoupling, in PAC2005 proceedings.

[7] G. Guignard, CERN Report No. 76-06, 1976.

[8] G. Guignard, Phys. Rev. E 51, p6104, 1995.

[9] H. Wiedemann, Particle Accelerator Physics II, Nonlinear and Higher-Order Beam Dynamics, SpringerVerlag, 1995.

[10] Y. Luo, Matrix perturbation approach to the weak linear coupling,BNL C-AD/AP Note 187, Jan., 2005, (unpublished).

[11] M. Billing, Cornell Report CBN 85-2 ( 1985 ).

[12] S. Peggs, IEEE Trans. Nucl. Sci. 30, 2460 (1983).

[13] Y. Luo, Phys. Rev. ST Accel. Beam 7, 124001 (2004). 


\section{Appendix}

\subsection{Hamiltonian perturbation approach [1]}

The perturbation Hamiltonian theory [7, 8, 9] gives the isolated linear difference coupling Hamiltonian as

$$
H_{2}=\left|C^{-}\right| \sqrt{a_{x} a_{y}} \cos \left(\Psi_{x}-\Psi_{y}+\Delta \cdot \varphi+\chi\right)
$$

where $\Delta$ is the uncoupled fractional tune split, $\Delta=Q_{x, 0}-Q_{y, 0}-p$. The coupling coefficient $C^{-}$is

$$
C^{-}=\left|C^{-}\right| e^{i \chi}=\frac{1}{2 \pi} \int_{0}^{L} \sqrt{\beta_{x} \beta_{y}} k_{s} e^{i\left[\Phi_{x}-\Phi_{y}-2 \pi \Delta \cdot s / L\right]} d l
$$

One can prove that

$$
C_{2}^{-}=C_{1}^{-} e^{i\left(\Delta \Phi_{x}-\Delta \Phi_{y}-\frac{2 \pi d s}{L} \cdot \Delta\right)},
$$

where $C_{1}^{-}$and $C_{2}^{-}$are the coupling coefficients calculated at point 1 and 2 , respectively. $\Delta \Phi_{x}$ and $\Delta \Phi_{y}$ are the unperturbed betatron phase advances, and $d s$ is the distance from point 1 to point 2 . Therefore, $\left|C^{-}\right|$is constant at different reference point. And knowing the coupling coefficient at one point, the coupling coefficient at other point can be calculated according to Eq. (12).

After some algebra calculations, the single particle motion is given by

$$
\left\{\begin{array}{l}
\left.\left.x(s)=\sqrt{2 \beta_{x}}\left\{a \cos \left[\Psi_{x}+(\nu-\Delta / 2) \varphi-\chi / 2\right)\right]+b \cos \left[\Psi_{x}-(\nu+\Delta / 2) \varphi-\chi / 2\right)\right]\right\} \\
\left.\left.y(s)=\sqrt{2 \beta_{y}}\left\{c \cos \left[\Psi_{y}+(\nu+\Delta / 2) \varphi+\chi / 2\right)\right]+d \cos \left[\Psi_{y}-(\nu-\Delta / 2) \varphi+\chi / 2\right)\right]\right\}
\end{array}\right.
$$

where

$$
\begin{gathered}
\nu=\frac{1}{2} \sqrt{\Delta^{2}+\left|C^{-}\right|^{2}} \\
\left\{\begin{array}{l}
\frac{c}{a}=\frac{\left|C^{-}\right|}{2 \nu+\Delta} \\
\frac{b}{d}=-\frac{\left|C^{-}\right|}{2 \nu+\Delta}
\end{array}\right.
\end{gathered}
$$

According to Eq. (13), the eigentunes are

$$
\left\{\begin{array}{c}
Q_{I}=Q_{x, 0}-\frac{1}{2} \Delta+\frac{1}{2} \sqrt{\Delta^{2}+\left|C^{-}\right|^{2}} \\
Q_{I I}=Q_{y, 0}+\frac{1}{2} \Delta-\frac{1}{2} \sqrt{\Delta^{2}+\left|C^{-}\right|^{2}}
\end{array}\right.
$$

The fractional eigentune split is

$$
\left|Q_{I}-Q_{I I}-p\right|=\sqrt{\Delta^{2}+\left|C^{-}\right|^{2}}
$$

Comparing Eq. (13) to Eq. (1),

$$
\begin{gathered}
\left\{\begin{array}{l}
R_{I}=\sqrt{\frac{\beta_{y}}{\beta_{x}}} \cdot \frac{\left|C^{-}\right|}{2 \nu+\Delta} \\
R_{I I}=\sqrt{\frac{\beta_{x}}{\beta_{y}}} \cdot \frac{\left|C^{-}\right|}{2 \nu+\Delta}
\end{array}\right. \\
\left\{\begin{array}{c}
\Delta \phi_{I}= \\
\Delta \phi_{I I}=\pi-\chi
\end{array}\right.
\end{gathered}
$$

And according to Eq. (18), when the coupling gets weaker, that is, $\left|C^{-}\right|$gets smaller, the amplitude ratios $R_{I}$ and $R_{I I}$ get smaller. When $\left|C^{-}\right|=0, R_{I}=R_{I I}=0$.

And it is noticed that $R_{I} R_{I I}$ is independent of local $\beta$ functions. Knowing the measured eigentune split and $R_{I}, R_{I I}$, the uncoupled tune split $\Delta$ and the coupling coefficient amplitude $\left|C^{-}\right|$can be determined,

$$
\begin{gathered}
R_{I} R_{I I}=\frac{\left|C^{-}\right|^{2}}{\left(\sqrt{\Delta^{2}+\left|C^{-}\right|^{2}}+\Delta\right)^{2}} \\
\left|C^{-}\right|=\frac{2 \sqrt{R_{I} R_{I I}}}{1+R_{I} R_{I I}}\left(Q_{I}-Q_{I I}-p\right), \\
\Delta=\frac{1-R_{I} R_{I I}}{1+R_{I} R_{I I}}\left(Q_{I}-Q_{I I}-p\right) .
\end{gathered}
$$




\subsection{Matrix perturbation approach [10]}

In the normalized coordinates are defined as

$$
\begin{gathered}
\overline{\mathbf{X}}=\mathbf{U}^{-1} \mathbf{X} \\
\mathbf{U}=\left(\begin{array}{cc}
\mathbf{U}_{I} & \mathbf{0} \\
\mathbf{0} & \mathbf{U}_{I I}
\end{array}\right), \\
\mathbf{U}_{i}=\left(\begin{array}{cc}
\sqrt{\beta_{i}} & 0 \\
\frac{-\alpha_{i}}{\sqrt{\beta_{i}}} & \frac{1}{\sqrt{\beta_{i}}}
\end{array}\right) .
\end{gathered}
$$

They also can be expressed with

$$
\begin{gathered}
\overline{\mathbf{X}}=\overline{\mathbf{V}}\left(\begin{array}{c}
\sqrt{2 J_{I}} \cos \Phi_{I} \\
-\sqrt{2 J_{I}} \sin \Phi_{I} \\
\sqrt{2 J_{I I}} \cos \Phi_{I I} \\
-\sqrt{2 J_{I I}} \sin \Phi_{I I}
\end{array}\right), \\
\overline{\mathbf{V}}=\left(\begin{array}{cc}
r \mathbf{I} & \overline{\mathbf{C}} \\
-\overline{\mathbf{C}}^{+} & r \mathbf{I}
\end{array}\right) .
\end{gathered}
$$

Under thin lens and weak coupling assumption, using the uncoupled Twiss parameters in $\mathbf{U}$, considering Eq. (26) and (23),

$$
\left\{\begin{array}{l}
x=r \sqrt{\beta_{x}} \sqrt{2 J_{I}} \cos \Phi_{I}+\bar{c}_{11} \sqrt{\beta_{x}} \sqrt{2 J_{I I}} \cos \Phi_{I I}-\bar{c}_{12} \sqrt{\beta_{x}} \sqrt{2 J_{I I}} \sin \Phi_{I I} \\
y=-\bar{c}_{22} \sqrt{\beta_{y}} \sqrt{2 J_{I}} \cos \Phi_{I}-\bar{c}_{12} \sqrt{\beta_{y}} \sqrt{2 J_{I}} \sin \Phi_{I}+r \sqrt{\beta_{y}} \sqrt{2 J_{I I}} \cos \Phi_{I I}
\end{array} .\right.
$$

Then the amplitude ratios and the phase differences are

$$
\begin{gathered}
\left\{\begin{array}{c}
R_{I}=\sqrt{\frac{\beta_{y}}{\beta_{x}}} \frac{\sqrt{\bar{c}_{22}^{2}+\bar{c}_{12}^{2}}}{r} \\
R_{I I}=\sqrt{\frac{\beta_{x}}{\beta_{y}}} \frac{\sqrt{\bar{c}_{11}^{2}+\bar{c}_{12}^{2}}}{r}
\end{array}\right. \\
\left\{\begin{array}{c}
\Delta \phi_{I, 0}=-\arctan \frac{\bar{c}_{12}}{\bar{c}_{22}} \\
\Delta \phi_{I I, 0}=\arctan \frac{\bar{c}_{12}}{\bar{c}_{11}}
\end{array}\right.
\end{gathered}
$$

For weak difference coupling, Under thin lens and weak coupling assumption, according to [10],

$$
\begin{aligned}
& |\operatorname{Tr}(\overline{\mathbf{A}}-\overline{\mathbf{B}})|=\left|2 \cos \left(2 \pi Q_{1}\right)-2 \cos \left(2 \pi Q_{2}\right)\right|=\sqrt{4\left(\cos 2 \pi \mu_{x}-\cos 2 \pi \mu_{y}\right)^{2}+4 \sin ^{2} \pi\left(\mu_{x}+\mu_{y}\right)\left|h_{-}\right|^{2}}, \\
& r=\sqrt{\frac{1}{2}+\frac{1}{2} \sqrt{\frac{\sin ^{2} \pi\left(\mu_{x}-\mu_{y}\right)}{\sin ^{2} \pi\left(\mu_{x}-\mu_{y}\right)+\frac{1}{4}\left|h_{-}\right|^{2}}}}, \\
& \overline{\mathbf{C}}=-\frac{\sin \pi\left(Q_{x, 0}+Q_{y, 0}\right)}{r \operatorname{Tr}(\overline{\mathbf{A}}-\overline{\mathbf{B}})}\left(\begin{array}{cc}
\operatorname{Re}\left\{h_{-}\right\} & \operatorname{Im}\left\{h_{-}\right\} \\
-\operatorname{Im}\left\{h_{-}\right\} & \operatorname{Re}\left\{h_{-}\right\}
\end{array}\right) \text {. } \\
& h_{-}=\sum_{j=1}^{N}\left(\sqrt{\beta_{x} \beta_{y}} k_{s} d l\right)_{j} e^{i\left[\pi\left(Q_{x, 0}-Q_{y, 0}\right)-\left(\phi_{x, j}-\phi_{y, j}\right)\right]} .
\end{aligned}
$$

The fractional eigen tune split is

$$
\left(Q_{I}-Q_{I I}-p\right)^{2}=\left(Q_{x, 0}-Q_{y, 0}-p\right)^{2}+\left(\frac{1}{2 \pi}\left|h_{-}\right|\right)^{2},
$$

which is equal to Eq. (17) from Hamiltonian perturbation approach. 
And considering Eq. (33), together with Eqs. (29) and (30),

$$
\begin{aligned}
& \left\{\begin{array}{rl}
R_{I} & =\sqrt{\frac{\beta_{y}}{\beta_{x}}} \frac{\sqrt{1-r^{2}}}{r} \\
R_{I I} & =\sqrt{\frac{\beta_{x}}{\beta_{y}}} \frac{\sqrt{1-r^{2}}}{r} \\
R_{I} R_{I I} & =\frac{1-r^{2}}{r^{2}}
\end{array},\right. \\
& \left\{\begin{array}{ccc}
\Delta \phi_{I, 0} & = & \pi-\chi_{h} \\
\Delta \phi_{I I, 0} & = & \chi_{h}
\end{array},\right.
\end{aligned}
$$

$\chi_{h}$ is the phase of $h_{-}, h_{-}=\left|h_{-}\right| e^{i \chi_{h}}$.

\section{3 $\quad$ Strict matrix approach [13]}

In action-angle parameterization, single particle motion is represented by

$$
\begin{gathered}
\left(\begin{array}{c}
x \\
x^{\prime} \\
y \\
y^{\prime}
\end{array}\right)=\mathbf{P} \cdot\left(\begin{array}{c}
\sqrt{2 J_{1}} \cos \Phi_{1} \\
-\sqrt{2 J_{1}} \sin \Phi_{1} \\
\sqrt{2 J_{2}} \cos \Phi_{2} \\
-\sqrt{2 J_{2}} \sin \Phi_{2}
\end{array}\right), \\
\mathbf{P}=\left(\begin{array}{cccc}
p_{11} & 0 & p_{13} & p_{14} \\
p_{21} & p_{22} & p_{23} & p_{24} \\
p_{31} & p_{32} & p_{33} & 0 \\
p_{41} & p_{42} & p_{43} & p_{44}
\end{array}\right),
\end{gathered}
$$

where $J_{I, I I}, \Phi_{I, I I}$ are the actions and betatron phases of the two eigenmodes.

Matrix $\mathbf{P}$ can be derived from the eigenvectors of the one-turn transfer map. And it also has tight connections to Twiss and coupling parameters defined in Edwards-Teng's linear coupling parameterization

$$
\mathbf{P}=\left(\begin{array}{cccc}
r \sqrt{\beta_{1}} & 0 & c_{11} \sqrt{\beta_{2}}-c_{12} \alpha_{2} / \sqrt{\beta_{2}} & c_{12} / \sqrt{\beta_{2}} \\
-\alpha_{1} r / \sqrt{\beta_{1}} & r / \sqrt{\beta_{1}} & c_{21} \sqrt{\beta_{2}}-c_{22} \alpha_{2} / \sqrt{\beta_{2}} & c_{22} / \sqrt{\beta_{2}} \\
-c_{12} \alpha_{1} / \sqrt{\beta_{1}}-c_{22} \sqrt{\beta_{1}} & c_{12} / \sqrt{\beta_{1}} & r \sqrt{\beta_{2}} & 0 \\
c_{11} \alpha_{1} / \sqrt{\beta_{1}}+c_{21} \sqrt{\beta_{1}} & -c_{11} / \sqrt{\beta_{1}} & -\alpha_{2} r / \sqrt{\beta_{2}} & r / \sqrt{\beta_{2}}
\end{array}\right) .
$$

In order to easily distinguish Twiss parameter $\gamma$, here we use $r$ to take place of the coupling parameter $\gamma$ in other literatures.

According to Eq. (38),

$$
\left\{\begin{array}{l}
x=p_{11} \sqrt{2 J_{1}} \cos \Phi_{1}+p_{13} \sqrt{2 J_{2}} \cos \Phi_{2}-p_{14} \sqrt{2 J_{2}} \sin \Phi_{2} \\
y=p_{31} \sqrt{2 J_{1}} \cos \Phi_{1}-p_{32} \sqrt{2 J_{1}} \sin \Phi_{1}+p_{33} \sqrt{2 J_{2}} \cos \Phi_{2}
\end{array} .\right.
$$

Therefore,

$$
\begin{aligned}
& \left\{\begin{array}{l}
R_{I}=\sqrt{p_{31}^{2}+p_{32}^{2}} / p_{11} \\
R_{I I}=\sqrt{p_{13}^{2}+p_{14}^{2}} / p_{33}
\end{array},\right. \\
& \left\{\begin{array}{rl}
\Delta \phi_{I} & =\arctan \left(p_{32} / p_{31}\right) \\
\Delta \phi_{I I} & =\arctan \left(p_{14} / p_{13}\right)
\end{array} .\right.
\end{aligned}
$$

According to Eq.( 40$), R_{I, I I}$ and $\Delta \phi_{I, I I}$ are expressed in Twiss and coupling parameters,

$$
\begin{gathered}
\left\{\begin{array}{c}
R_{I}=\sqrt{\beta_{1} c_{22}^{2}+2 \alpha_{1} c_{22} c_{12}+\gamma_{1} c_{12}^{2}} /\left(r \sqrt{\beta_{1}}\right) \\
R_{I I}=\sqrt{\beta_{2} c_{11}^{2}-2 \alpha_{2} c_{11} c_{12}+\gamma_{2} c_{12}^{2}} /\left(r \sqrt{\beta_{2}}\right)
\end{array}\right. \\
\left\{\begin{array}{c}
\Delta \phi_{I}=\arctan \left(-c_{12} /\left(\alpha_{1} c_{12}+\beta_{1} c_{22}\right)\right) \\
\Delta \phi_{I I}=\arctan \left(c_{12} /\left(-\alpha_{2} c_{12}+\beta_{2} c_{11}\right)\right)
\end{array}\right.
\end{gathered}
$$

where we define $\gamma_{1}=\left(1+\alpha_{1}^{2}\right) / \beta_{1}, \gamma_{2}=\left(1+\alpha_{2}^{2}\right) / \beta_{2}$. 